\title{
Voltage-biased superconducting transition-edge bolometer with strong electrothermal feedback operated at $370 \mathrm{mK}$
}

\author{
Shih-Fu Lee, J an M. Gildemeister, Warren Holmes, Adrian T. Lee, and
}

Paul L. Richards

\begin{abstract}
We present an experimental study of a composite voltage-biased superconducting bolometer (VSB). The tested VSB consists of a Ti-film superconducting thermometer $\left(T_{C} \sim 375 \mathrm{mK}\right)$ on a Si substrate suspended by $\mathrm{NbTi}$ superconducting leads. A resistor attached to the substrate provides calibrated heat input into the bolometer. The current through the bolometer is measured with a superconducting quantum interference device ammeter. Strong negative electrothermal feedback fixes the bol ometer temperature at $T_{C}$ and reduces the measured response time from $2.6 \mathrm{~s}$ to $13 \mathrm{~ms}$. As predicted, the measured current responsivity of the bolometer is equal to the inverse of the bias voltage. A noise equivalent power of $5 \times$ $10^{-17} \mathrm{~W} / \mathrm{V} \mathrm{Hz}$ was measured for a thermal conductance $\mathrm{G} \sim 4.7 \times 10^{-10} \mathrm{~W} / \mathrm{K}$, which is consistent with the expected thermal noise. Excess noise was observed for bias conditions for which the electrothermal feedback strength was close to maximum. (c) 1998 Optical Society of America
\end{abstract}

OCIS codes: $120.0120,040.3060$.

\section{Introduction}

Bolometers are sensitive, broadband millimeterwave and infrared detectors used extensively in astronomical observations and in laboratory spectroscopy. ${ }^{1}$ In many applications, bol ometer performance is limited by a trade-off between speed and sensitivity. For example, maximum sensitivity for low-background astronomical measurements such as balloonborne observations of the cosmic microwave background ${ }^{2}$ is usually achieved at the expense of bolometer time constants. In applications in which F ourier spectrometers are used, a fast bol ometer with a linear response over large changes in radiation power is often desired. With conventional bolometers, these properties are usually achieved at the expense of sensitivity. Recently, the voltage-biased superconducting bolometer (VSB) was introduced. ${ }^{3}$ This device potentially has a much better combination of speed and sensitivity than technologies currently in use. ${ }^{4}$ The large $d(\log R) / d(\log T)$ of the

The authors are with the Department of Physics and the Materials Sciences Division, Lawrence Berkeley National Laboratory, University of California, Berkeley, California 94720-7300. A. T. Lee is also with the Center for Particle Astrophysics.

Received 17 November 1997; revised manuscript received 2 February 1998.

0003-6935/98/163391-07\$15.00/0

(C) 1998 Optical Society of America superconducting transition results in a strong negative electrothermal feedback (ETF) effect ${ }^{5}$ that fixes the bolometer temperature at $T_{C}$ and reduces the bolometer response time by orders of magnitude less than the intrinsic time constant. In this strong ETF regime, the current responsivity of the VSB is equal to the inverse of the bias vol tage over a wide range of signal power. Unlike a conventional bolometer in which the output is proportional to the temperature change, the VSB is a null detector whose output is the feedback current needed to hold the temperature constant. Because of the large $d(\log R) / d(\log T)$ of the VSB and the low noise of superconducting quantum interference device (SQUID) amplifiers, the noiseequivalent power (NEP) can approach the limit set with thermal fluctuations.

In this paper we present experimental data on a composite VSB that consists of a Ti-film superconducting thermometer $\left(T_{c} \sim 375 \mathrm{mK}\right)$ on a Si substrate suspended by $\mathrm{NbTi}$ superconducting wires that provide both electrical connection to the thermometer and a thermal link with conductance $G \sim 4.7 \times 10^{-10}$ $\mathrm{W} / \mathrm{K}$ to an $\sim 270-\mathrm{mK}$ cold stage. The current through the bolometer is read out with a SQUID ammeter. Earlier research ${ }^{4}$ presented measurements of a VSB operated at $\sim 100 \mathrm{mK}$ for which thermal isolation was achieved with electron-phonon decoupling. The research presented here demonstrates that the benefits of strong ETF can berealized at a temperature appropriate for ${ }^{3} \mathrm{He}$ refrigerators 
and with a composite bol ometer structure incorporating a conventional weak thermal link. The presentation of the theory has been significantly improved compared with the earlier research, and a detailed comparison between theory and experiment has been made for the first time to our knowledge. In the following sections, we discuss the theory of the VSB, the design and fabrication of the bolometer, the experimental apparatus, and the measurements of response time, responsivity, and noise.

\section{Voltage-Biased Superconducting Bolometer Theory}

The operation of a VSB can be illustrated by a simple model: a bolometer with heat capacity $C$ is connected to $a$ heat sink at temperature $T_{0}$ with an average thermal conductance $\bar{G}$. The bolometer is heated up to its operating temperature $T$ by background power $\mathrm{P}$ and bias power $\mathrm{V}_{\mathrm{b}}{ }^{2} / \mathrm{R}$, where $\mathrm{V}_{\mathrm{b}}$ is the bias voltage and $R$ is the resistance of the transition-edge thermometer. When the substrate absorbs a timevarying signal power $\delta P \exp (i \omega t)$, the resulting temperature rise, $\delta \mathrm{T} \exp (\mathrm{i} \omega \mathrm{t})(\delta \mathrm{T} \ll \mathrm{T})$, causes a decrease in the bias power. The power flow for this model is given by ${ }^{1}$

$$
\begin{aligned}
P & +\delta P \exp (i \omega t)+\frac{V_{b}^{2}}{R}-\frac{V_{b}^{2}}{R^{2}} \frac{d R}{d T} \delta T \exp (i \omega t) \\
& =\bar{G}\left(T-T_{0}\right)+G \delta T \exp (i \omega t)+i \omega C \delta T \exp (i \omega t),
\end{aligned}
$$

where $\mathrm{G}=\mathrm{dP}_{\text {total }} / \mathrm{dT}$ is the differential thermal conductance of the weak link at temperature T. Wecan separate Eq. (1) into the time-independent term P + $\mathrm{V}_{\mathrm{b}}{ }^{2} / \mathrm{R}=\overline{\mathrm{G}}\left(\mathrm{T}-\mathrm{T}_{0}\right)$ and the time-dependent term

$$
\delta P \exp (i \omega t)=\left(\frac{P_{b}}{T} \alpha+G+i \omega C\right) \delta T \exp (i \omega t),
$$

where $\alpha=\mathrm{d}(\log \mathrm{R}) / \mathrm{d}(\log \mathrm{T})$ is a dimensionless parameter describing the sharpness of the transition. The first term gives the negative ETF; when it is large, it reduces the temperature response $\delta T$ because the bias power change compensates the change of signal power in the bolometer. From Eq. (2) we define an effective complex thermal conductance, ${ }^{6}$ which controls the temperature response of VSB to the incident signal power

$$
G_{\text {eff }}=\frac{P_{b}}{T} \alpha+G+i \omega C .
$$

It is clear that the effective thermal conductance of the VSB is increased by the effect of negative ETF. We define a loop gain $\mathscr{L}(\omega)$ for ETF from the formula

$$
\mathscr{L}(\omega)\left(\delta \mathrm{P}+\delta \mathrm{P}_{\mathrm{b}}\right)=\mathscr{L}(\omega) \delta \mathrm{P}_{\text {total }}=-\delta \mathrm{P}_{\mathrm{b}},
$$

which is analogous to that used for electronic feedback circuits. ${ }^{7}$ This gain is frequency dependent and is given by the ratio of the terms in $\mathrm{G}_{\text {eff, }}$,

$$
\mathscr{L}(\omega)=\frac{\mathrm{P}_{\mathrm{b}} \alpha}{\mathrm{GT}\left(1+\mathrm{i} \omega \tau_{0}\right)}=\frac{\mathscr{L}}{1+\mathrm{i} \omega \tau_{0}},
$$

where $\mathscr{L}$ is the dc gain. ${ }^{3}$ The gain rolls off at $\omega>$ $1 / \tau_{0}=\mathrm{G} / \mathrm{C}$.

At frequencies $\omega \ll 1 / \tau_{0}$, the temperature change $\delta \mathrm{P} / \mathrm{G}(\mathscr{L}+1)$ is reduced by a factor of $\mathscr{L}+1$ compared with that without ETF. The VSB output is the change of bias current and its current responsivity is defined as $S_{i} \equiv \delta l / \delta P . \quad$ From Eq. (2), ${ }^{3}$

$$
\mathrm{S}_{\mathrm{i}}=\frac{-1}{\mathrm{~V}_{\mathrm{b}}} \frac{\mathscr{L}}{(\mathscr{L}+1)} \frac{1}{(1+\mathrm{i} \omega \tau)}
$$

where $\tau=\tau_{0} /(\mathscr{L}+1)$ is an effective time constant. Two features of the VSB are immediately apparent from this expression. In the limit $\mathscr{L} \gg 1$ with $\omega \ll$ $1 / \tau$, the responsivity $S_{i} \sim-1 / V_{b}$ is simply given by the bias voltage. Also, the frequency response of the VSB rolls off at the higher frequency $1 / \tau$ rather than $1 / \tau_{0}$.

Since the mean-square fluctuations from uncorrelated sources can be added in quadrature, the NEP for a VSB can be written

$$
\begin{aligned}
N E P^{2}= & N E P_{\text {photon }}{ }^{2}+\gamma 4 k T^{2} G \\
& +\frac{4 k T / R}{\left|S_{i}\right|^{2}}\left(\frac{\tau}{\tau_{0}}\right)^{2}\left(\frac{1+\omega^{2} \tau_{0}{ }^{2}}{1+\omega^{2} \tau^{2}}\right) \\
& +\frac{\mathrm{i}_{\text {SQUID }}{ }^{2}}{\left|S_{i}\right|^{2}}+N E P_{\text {excess }}{ }^{2},
\end{aligned}
$$

where the terms describe photon noise, thermal fluctuation noise, J ohnson noise, amplifier noise, and excess low-frequency noise (often $1 / f$ ).

In our experiments, photon noise does not contribute since we use heater power to simulate optical power in the bolometer that is operated in the dark. Two types of fundamental thermal noise set limits to the performance of the bolometer: thermal fluctuation noise and J ohnson noise. Thermal fluctuation noise is caused by the random propagation of energy carriers between the thermometer and the heat sink. The fluctuations of power flow have a spectral density $N E P_{\text {thermal }}{ }^{2}=\gamma 4 k T^{2} G$, where $\gamma$ is a factor less than unity representing an adjustment to the thermal fluctuation noise for the gradient in temperature along the thermal link. ${ }^{8}$ This factor is calculated by Mather assuming $\mathrm{G} \propto \mathrm{nT}^{\mathrm{n}-1}$ and is approximately $1-(1+n / 2) t+(2+n)(2+3 n) t^{2} / 12$, where $t=1-$ $T_{0} / T$. For a constant $R$, J ohnson noise generates a fluctuating current in a closed circuit with a spectral density $\mathrm{i}_{\mathrm{n}}{ }^{2}=4 \mathrm{kT} / \mathrm{R}$. However, Mather points out that these current fluctuations are reduced by the large dynami c resistance 8 of a bolometer in the strong ETF limit. As shown in Eq. (7), the contribution from $\mathrm{J}$ ohnson noise is reduced by $1 /(\mathscr{L}+1)$ for $\omega<$ $1 / \tau_{0}$ and gradually increases with frequency until it reaches its usual value for $\omega>1 / \tau$. The $i_{\text {souid }}{ }^{2}$ term in Eq. (7) is the noise current of the SQUID. Excess noise for a VSB may have contributions from various sources, such as fluctuations in $\mathrm{T}_{0}$, microphonics, contact shot noise, ${ }^{8}$ resistivity fluctuations, ${ }^{9}$ and superconducting flux noise. ${ }^{10}$

For direct comparison with measurements, it is 


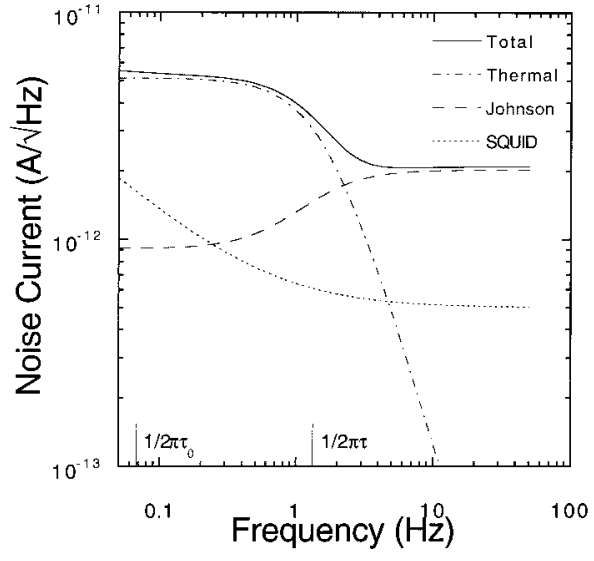

Fig. 1. Predicted noise current of the VSB tested, referred to the input of the SQUID. The total noise current is obtained when J ohnson, thermal fluctuation (phonon) and SQUID noise are added in quadrature with Eq. (8). The input parameters for the plots $\operatorname{are~}_{\mathrm{b}}=8.8 \mu \mathrm{V}, \overline{\mathrm{G}}=3.5 \times 10^{-10} \mathrm{~W} / \mathrm{K}, \mathrm{R}=5 \Omega, \mathrm{T}=375 \mathrm{mK}, \mathrm{T}_{0}$ $=270 \mathrm{mK}, \mathrm{dR} / \mathrm{dT}=2.5 \times 10^{3} \Omega / \mathrm{K}, \tau_{0}=2.6 \mathrm{~s}, \tau=0.13 \mathrm{~s}$, and $\mathrm{i}_{\text {SQuID }}{ }^{2}=\left(5 \times 10^{-13}\right)^{2}+\left(4 \times 10^{-13}\right)^{2} / \mathrm{f} \mathrm{A} / \sqrt{ } \mathrm{Hz}$ at $\mathrm{f}>0.1 \mathrm{~Hz}$. These parameters correspond to a relatively low gain $\mathscr{L}=20$, which gave the best experimental noise performance.

useful to rewrite the noise of a VSB in terms of noise current referred to the input of the SQUID,

$$
\begin{aligned}
\mathrm{I}_{\mathrm{n}}{ }^{2}= & \left|\mathrm{S}_{\mathrm{i}}\right|^{2} \gamma 4 \mathrm{kT} \mathrm{T}^{2} \mathrm{G}+\frac{4 \mathrm{kT}}{\mathrm{R}}\left(\frac{\tau}{\tau_{0}}\right)^{2}\left(\frac{1+\omega^{2} \tau_{0}^{2}}{1+\omega^{2} \tau^{2}}\right) \\
& +\mathrm{i}_{\text {SQUID }}{ }^{2}+\mathrm{i}_{\text {excess }}{ }^{2} .
\end{aligned}
$$

In Fig. 1, a theoretical noise spectrum of the VSB under test is computed with Eq. (8) with no excess noise. The input parameters are taken from the VSB and the SQUID used in this study, and $S_{i}(\omega)$ is calculated from Eq. (6). The plot in Fig. 1 demonstrates qualitative features of the J ohnson and the thermal fluctuation noise predicted for a VSB. The predicted total noise current can be compared with experimental results.

\section{Bolometer Fabrication and Operation}

The measured bolometer has a composite structure, including a thermometer, electrical leads, and suspension wires (Fig. 2). The thermometer is a 40-nmthick film of Ti sputtered on a 0.3-mm-thick Si wafer that was then diced to $1.5 \mathrm{~mm} \times 1.5 \mathrm{~mm}$ with a diamond saw. No special effort was made to reduce the heat capacity. The thermal mass of the Si substrate, for example, could have been reduced by nearly 1 order of magnitude. Electrical contact to the Ti film is provided by $1.5 \mathrm{~mm} \times 0.2 \mathrm{~mm}$ superconducting $\mathrm{Al}-\mathrm{Au}$ bilayer contacts to obtain a uniform current distribution. The 200-nm Au film is evaporated on top of the 80-nm Al film without breaking vacuum to provide low-resistance contacts with the electrical leads. Both the suspension wires and the electrical leads use $12.5-\mu \mathrm{m}$-diameter $\mathrm{NbTi}$ wires. The mechanical suspension consists of two parallel $\mathrm{NbTi}$ wires tensioned and soldered onto $\mathrm{Cu}$

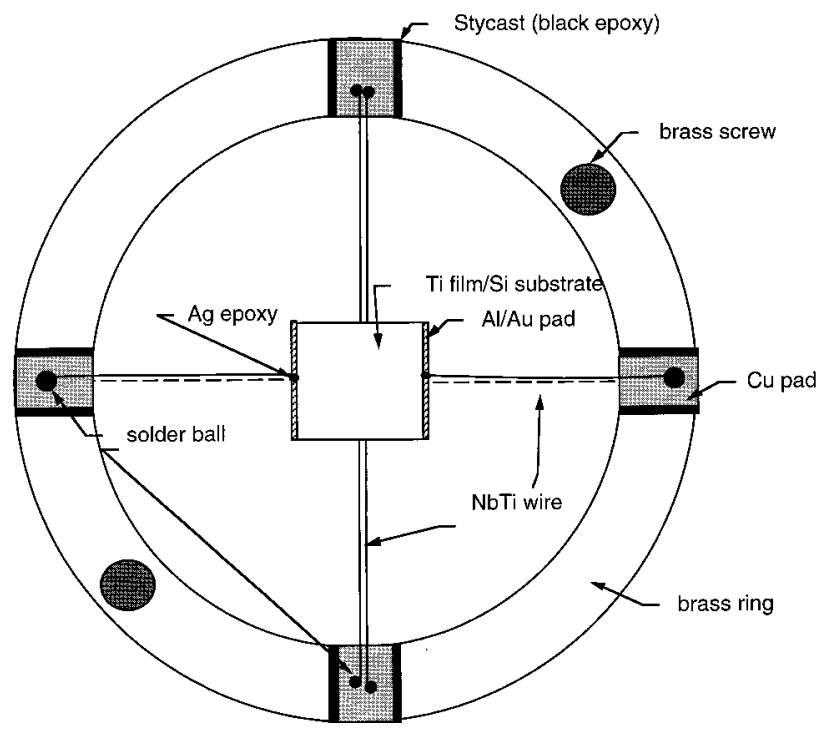

Fig. 2. Diagram of the VSB tested. A NiCr heater chip is attached to the back of the substrate. The bolometer substrate is $1.5 \mathrm{~mm} \times 1.5 \mathrm{~mm}$.

contact pads that are epoxied ${ }^{11}$ to the $\mathrm{Cu}$ bolometer ring as shown in Fig. 3. The bolometer substrate is attached to the middle of the suspension wires with epoxy. The electrical leads are attached to the $\mathrm{Au}$ pads with Ag epoxy. ${ }^{12}$ By chemical etching everywhere except at the ends where solder or Ag-epoxy connections are made, we removed the $2-\mu \mathrm{m}$ Cu cladding on the wires. The thermal conductance is dominated by the two electrical leads that have $\sim 5$ $\mathrm{mm}$-long etched regions to give $\overline{\mathrm{G}} \sim 10^{-10} \mathrm{~W} / \mathrm{K}$. On the reverse side of the bol ometer substrate, a 7.18- $\Omega$ $\mathrm{NiCr}$ heater chip is attached to the bolometer with epoxy. The heater chip is current biased, and the power generated simulates optical power incident on the bolometer. A Bi film could be deposited on this side of the substrate to absorb radiation. ${ }^{1}$

The test cryostat has a charcoal-pumped ${ }^{3} \mathrm{He}$ re frigerator with a base temperature of $\sim 260 \mathrm{mK}$ attached to a superfluid ${ }^{4} \mathrm{He}$ cold plate. ${ }^{13}$ We varied the temperature of the refrigerator by heating the charcoal sorption pump. A sample stage at $\sim 270$

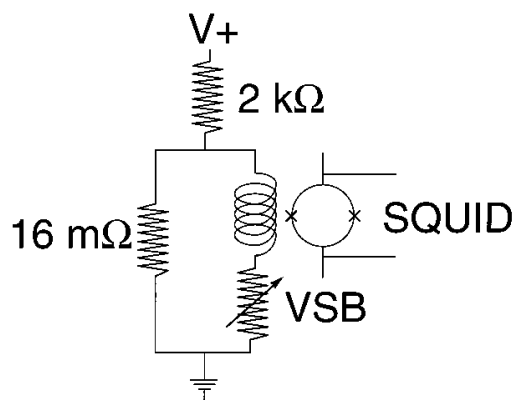

Fig. 3. Voltage-biased bolometer circuit with SQUID read-out amplifier. The VSB operates on the superconducting transition with $2 \leq R \leq 5 \Omega$. The reactance of the SQUID input coil is negligible. 
$\mathrm{mK}$ is connected to the ${ }^{3} \mathrm{He}$ refrigerator by a $\mathrm{Cu}$ wire with a thermal conductance $\sim 10^{-4} \mathrm{~W} / \mathrm{K}$, giving a 10-s time constant. This low-pass filter, with a cutoff frequency of $\sim 10^{-2} \mathrm{~Hz}$, reduces the effects of temperature fluctuations in the ${ }^{3} \mathrm{He}$ refrigerator in bolometer operation. The bolometer is shielded from ambient infrared radiation by a $\mathrm{Cu}$ enclosure, and the bolometer ring is clamped to the samplestage by brass screws. The inside of the $\mathrm{Cu}$ enclosure is painted with infrared absorbing paint made from Stycast epoxy, lampblack, and glass beads. ${ }^{14}$ This $\mathrm{Cu}$ encl osure is designed to reduce the optical load on the bolometer to a negligible level. The transition temperature of the bolometer in this enclosure was measured to be within $5 \mathrm{mK}$ of the transition temperature of several witness samples from the same wafer when these were heat sunk directly to the cold stage. This result implies that $<5 \%$ of the power needed to bias the bolometer in the transition is optical. The vol tage-bias circuit is illustrated in Fig. 3, which consists of a SQUID input coil in series with the bolometer and a 16.2-m $\Omega$ shunt resistor in parallel with the bolometer and the SQUID coil. The bol ometer bias vol tage is obtained when this circuit is current biased. The components of this circuit are mounted on the sample stage, except for the Quantum Design SQUID ${ }^{15}$ with its input coil, which is mounted on the ${ }^{4} \mathrm{Hecold}$ plate and is connected to the bias circuit by a twisted pair of NbTi wires. A Quantum Design SQUID controller ${ }^{15}$ was used.

We used heater power to simulate the background optical power and the signal power for measurements of responsivity, time constant, and noise. The bolometer was typically operated with a resistance higher than $1 \Omega$. For time-constant measurements, we applied a step in heater power and read out the current response of the VSB from the SQUID. The timetraces of the current response and the noise data were recorded with a signal analyzer. ${ }^{16}$

\section{Results}

The resistive transition of the bolometer was measured for bias voltages of 1 and $3 \mathrm{nV}$ for both the warming and the cooling directions. All measurements yielded curves similar to that in Fig. 4, indicating that negative ETF does not affect the measured $R(T)$ for these voltages. The $10-90 \%$ transition width is $\sim 2 \mathrm{mK}$, and there is a $0.5-\Omega$ residual resistance, which presumably comes from the contacts. The largest value of $\alpha$ for this Ti film is $\sim 1000$ at $\mathrm{R}=1.5 \Omega$.

The operating point on the resistive transition moves monotonically to higher resistance with increase of either bias power or heater power. Figure 5(a) shows that the total power $P_{\text {total }}$, which is the sum of bias power $\mathrm{P}_{\mathrm{b}}$ and heater power $\mathrm{P}_{\text {heater, }}$ re mains essentially constant for operating points that correspond to the steep portion of the transition. At a bias voltage of $5.24 \mu \mathrm{V}$, the deviation from linearity of $\delta \mathrm{P}_{\text {total }} / \mathrm{P}_{\text {total }}$ is less than $1 \%$ over a power range of $\sim 30 \mathrm{pW}$. Since the loop gain of the VSB is given by $\mathscr{L}=-\delta \mathrm{P}_{\mathrm{b}} / \delta \mathrm{P}_{\text {total }}$, wherever $\mathrm{P}_{\text {total }}$ is constant, the

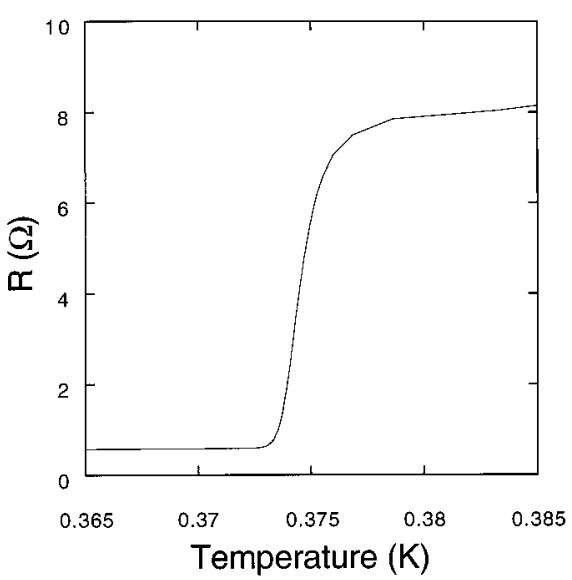

Fig. 4. Measured temperature dependence of the thermometer resistance. The largest value of $\alpha$ observed with this film is $\sim 1000$.

gain $\mathscr{L}$ is large and the responsivity is expected to be close to the reciprocal of the bias voltage as described by Eq. (6). In Fig. 5(b) we see that the bolometer current decreases linearly as $\mathrm{P}_{\text {heater }}$ increases when the VSB is biased on the transition and its slope, $\mathrm{dl} / \mathrm{dP}_{\text {heater, }}$, is equal to $-1 / \mathrm{V}_{\mathrm{b}}$ within $1 \%$ over a large range of power. In an actual application, the bolometer parameters would be adjusted so that the background infrared power biases the bolometer into the linear range that begins at $\sim 8 \mathrm{pW}$ in this example. The response to the signal and to any increase in background would be strictly linear. Note that, in this example, the power range where the VSB response is linear is 2 orders of magnitude larger than what could be achieved by a neutron-transmutationdoped Ge bolometer with similar $\bar{G}$ and bias power for which a criterion of a $1 \%$ responsivity change is used. We see in Fig. 6 that the product of the responsivity

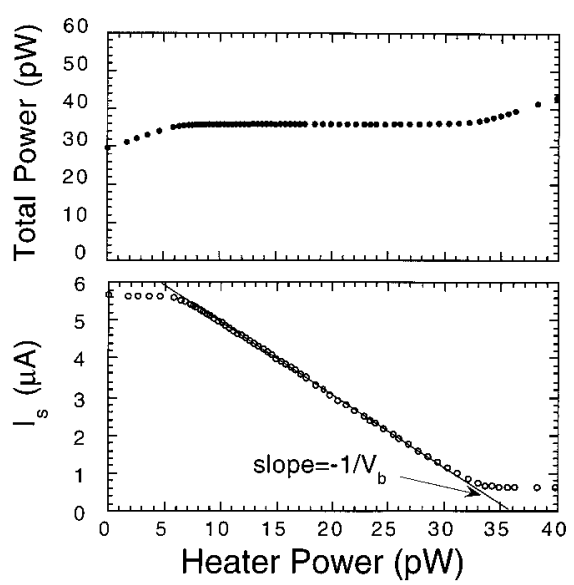

Fig. 5. Total power and current response measured with $V_{b}=5.4$ $\mu \mathrm{V}$. (a) The total power dissipated in the VSB is roughly constant and equal to $36 \mathrm{pW}$ for a wide range of heater power (8 pW $\leq$ $\Delta \mathrm{P}_{\text {heater }} \leq 33 \mathrm{pW}$ ) that corresponds to the steep part of the superconducting transition where the ETF is strong. (b) The VSB has a linear response to changes of heater power in the strong ETF regime, and the responsivity is given by $S_{i}=-1 / V_{b}$. 


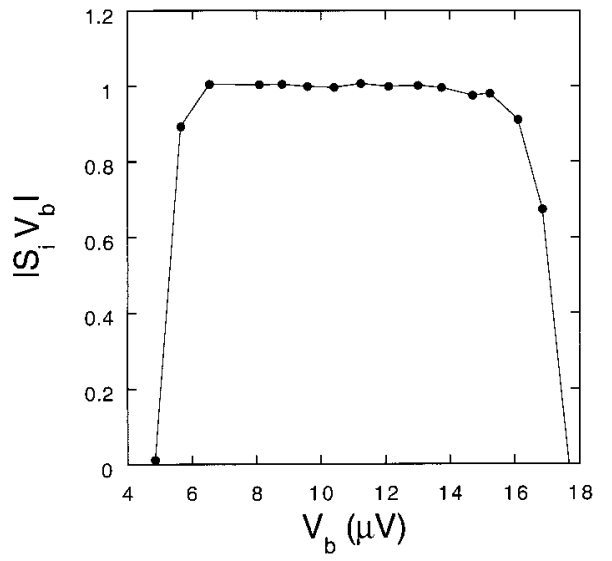

Fig. 6. Product of VSB responsivity and bias voltage $S_{i} V_{b}$ is constant over the range of bias voltage that corresponds to the steep part of the transition.

and the bias voltage is constant over the range of bias voltage where the VSB is biased on the transition.

The response of the VSB to a step in heater power is presented in Fig. 7 for a bias point low in the transition, where $\mathscr{L}$ is maximized and for a bias point above the transition where $\mathscr{L} \ll 1$. For the measurement with $\mathscr{L} \ll 1$, we obtain the intrinsic time constant $\mathrm{C} / \mathrm{G}$, which we find to be $2.6 \mathrm{~s}$. The time constant for the maximum $\mathscr{L}$ is $13 \mathrm{~ms}$ giving a factor of 200 decrease in response time.

It is difficult to determine $\mathscr{L}$ precisely for each measured $\tau$. Since $d R / d T$ is current dependent, especially at the lower end of the transition, it is not correct to derive $\mathscr{L}$ from values of $\alpha$ determined from the data in Fig. 4, which were measured in the lowcurrent limit. Therefore we measured $\delta \mathrm{P}_{\mathrm{b}}$ and $\delta \mathrm{P}_{\text {to }}-$ tal for each point by changing heater power, and we computed $\mathscr{L}=\delta \mathrm{P}_{\mathrm{b}} / \delta \mathrm{P}_{\text {total }}$ from Eq. (4). However, since the change of total power is very insensitive to the operation point in the strong ETF regime, errors

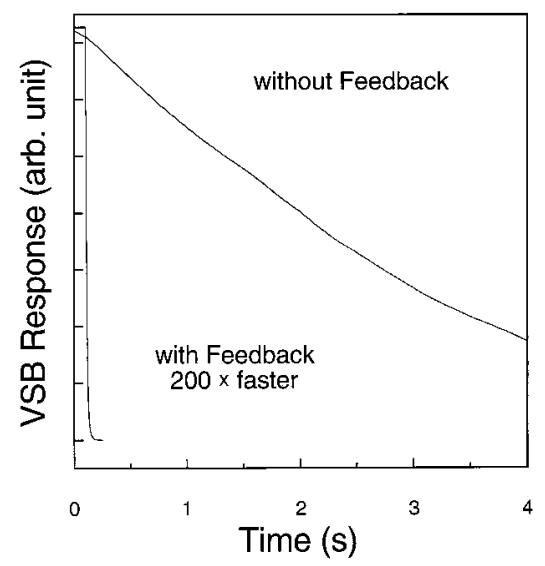

Fig. 7. Response of the VSB to a step in heater power. The data with negligible feedback is obtained when the VSB is operated above the transition where $\mathscr{L} \ll 1$, giving an intrinsic time constant $\tau_{0}=2.6 \mathrm{~s}$. A time constant of 13 ms was observed with $\mathscr{L} \sim$ 170.

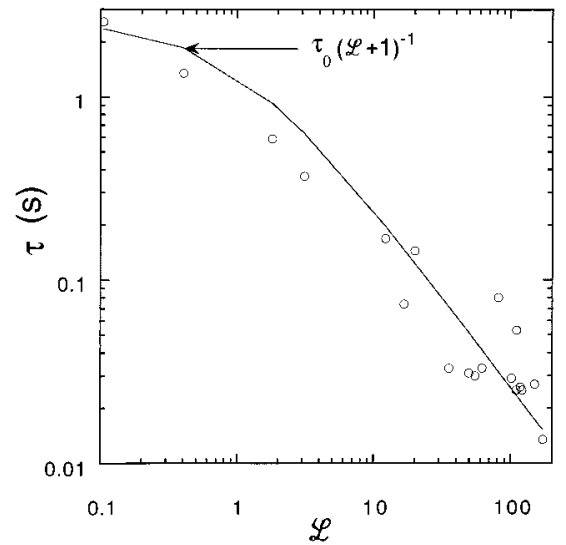

Fig. 8. Measured effective time constant $\tau$ as a function of gain $\mathscr{L}$. The curve gives the predicted dependence of $\tau=\tau_{0} /(\mathscr{L}+1)$, with $\tau_{0}=2.6 \mathrm{~s}$.

in determining $\mathscr{L}$ are as large as a factor 2 . In Fig. 8 we pl ot the measured $\tau$ versus $\mathscr{L}$ in comparison with the calculation of $\tau_{0} /(\mathscr{L}+1)$ from Eq. (6). The open circles are the measured data and the solid curve is calculated assuming $\tau_{0}=2.6 \mathrm{~s}$. Here we determined $\tau$ by fitting the time response data as shown in Fig. 7 to a single time-constant exponential function. The time-constant data follow the calculated curve over the entire range of $\mathscr{L}$, although the large uncertainty in $\mathscr{L}$ produces a large scatter.

The thermal conductance of the tested VSB can be extracted from $\bar{G}=P_{b}\left(T_{0}\right) /\left(T_{c}-T_{0}\right)$ and $G\left(T_{c}\right)=$ $\mathrm{nG} / \Sigma_{0}^{\mathrm{n}}\left(\mathrm{T}_{0} / \mathrm{T}\right)^{\mathrm{i}} .4 \quad$ From the results shown in Fig. 4 and in Fig. $5(\mathrm{a})$, we obtained $\overline{\mathrm{G}} \sim 3.5 \times 10^{-10} \mathrm{~W} / \mathrm{K}$ for the tested VSB. For NbTi wires, $\mathrm{n} \sim 3 .{ }^{17}$ We calculate $\mathrm{G}\left(\mathrm{T}_{\mathrm{c}}\right) \sim 4.7 \times 10^{-10} \mathrm{~W} / \mathrm{K}, \sqrt{\gamma} \sim 0.8$, and therefore $\mathrm{NEP}_{\text {thermal }} \sim 5 \times 10^{-17} \mathrm{~W} / \mathrm{V} \mathrm{Hz}$. At frequencies below $1 / 2 \pi \tau$, the calculated thermal noise current referred to the SQUID input is $5 \times 10^{-5} / \mathrm{V}_{\mathrm{b}}$ $\mathrm{pA} / \sqrt{ } \mathrm{Hz}$, where $\mathrm{V}_{\mathrm{b}}$ is the bias vol tage. The ohnson noise is 4.6/ $\sqrt{\mathrm{R}} \mathrm{pA} / \sqrt{\mathrm{Hz}}$ for $\mathrm{f}>1 / 2 \pi \tau$. The current noise of the SQUID is $\mathrm{i}_{\text {SQUID }} \sim 0.5 \mathrm{pA} / \sqrt{ } \mathrm{Hz}$ with a low-frequency noise corner of $0.5 \mathrm{~Hz}$.

We measured the VSB noise shown in Fig. 9 for $\mathrm{R}=2,3.5$, and $5 \Omega$ with the bias voltage $\mathrm{V}_{\mathrm{b}}=8.8 \mu \mathrm{V}$ by varying the heater power. The values of $\mathscr{L}$ corresponding to these operating parameters are roughly 150,80 , and 20 . Figure 1 shows that, in the absence of excess noise, the noise for $f<1 / 2 \pi \tau$ should be dominated by thermal fluctuations that give $\sim 5.7$ $\mathrm{pA} / \sqrt{ } \mathrm{Hz}$ for all R. The noise for $\mathrm{f}>1 / 2 \pi \tau$ is dominated by J ohnson noise, which gives 3.2, 2.4, and 2 $\mathrm{pA} / \sqrt{ } \mathrm{Hz}$ for resistances of $2,3.5$, and $5 \Omega$, respectively. The measured noise at $5 \Omega$, where the gain $\mathscr{L}$ $\approx 20$, agrees with the calculated thermal fluctuation noise and J ohnson noise for frequencies greater than $0.6 \mathrm{~Hz}$. Excess noise is seen for frequencies less than $0.6 \mathrm{~Hz}$, which has a frequency dependence close to $1 / f$ for all values of $R$. Possible candidates include microphonics from the suspension and the electrical wires, resistivity fluctuations of the superconducting $\mathrm{Ti}$ film, ${ }^{9}$ and superconducting flux 


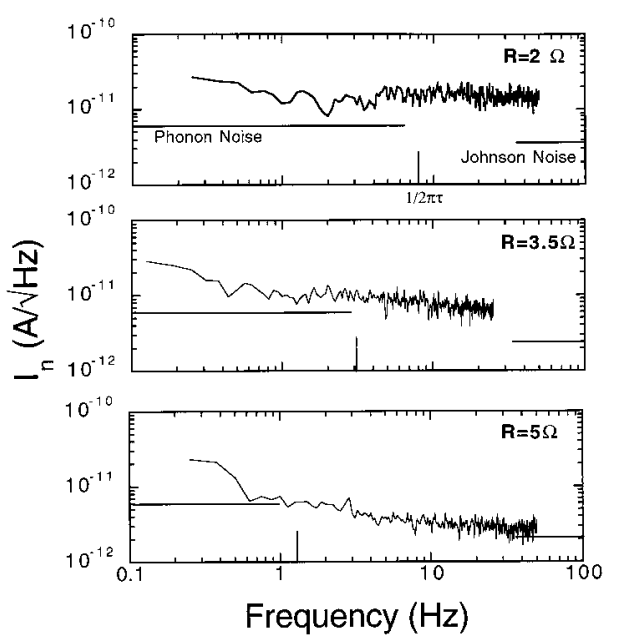

Fig. 9. Measured noise spectra for $V_{b}=8.8 \mu \mathrm{V}$ and $\mathrm{R}=2,3.5$, and $5 \Omega$. The operating point on the resistive transition is set when the heater power is changed. The estimated thermal fluctuation noise and J ohnson noise are indicated for each plot. The noise is predicted to approach thermal fluctuation noise for $\mathrm{f}<$ $1 / 2 \pi \tau$ and $\mathrm{J}$ ohnson noise for $f>1 / 2 \pi \tau$. Except for $1 / \mathrm{f}$ noise for $\mathrm{f}<0.6 \mathrm{~Hz}$, the data for $\mathrm{R}=5 \Omega$ (where $\mathscr{L}=20$ ) agree with the theory plotted in Fig. 1. Excess broadband noise is seen for $\mathrm{R}=$ 3.5 and $2 \Omega$ where $\mathscr{L} \sim 80$ and 150 .

motion. ${ }^{10}$ We examined the effect of microphonics by mounting an electromagnetic driver on the cryostat, which was driven from a white-noise source at several power levels. It was found that the threshold for increasing the noise at low frequencies with the driver was very low. Ambient acoustic energy may cause mechanical vibration of the bolometer giving a heat input that varies at low frequencies. This mechanism has been observed for many bolometers constructed in a similar manner.

In addition to the excess low-frequency noise, we see excess white noise that increases as the VSB resistance decreases and a broad peak that occurs roughly at $1 / 2 \pi \tau$. The broad peak in the noise spectra may be understood from the effects of a finite, internal time constant on the ETF. The model of VSB operation described above assumes no time delay in reaching internal thermal equilibrium after changes in the heater, bias, or noise power. However, with large $\mathscr{L}$, the bol ometer time constant $\tau$ may become comparable with internal time constants that then should not be neglected. We have explored models in which there are time delays between these power inputs. In general, the detector responsivity is then a more complicated function of frequency than is given in Eq. (6). In particular, if there is a delay between a thermal fluctuation in theinput power and the response of the superconducting film, a broad peak appears near $1 / 2 \pi \tau$ in responsivity and thus in the output noise power spectrum. This effect, which is illustrated in Fig. 10, is not excess noise but rather an underestimate of the responsivity for frequencies near $1 / 2 \pi \tau$. The peak in the simulation is sharper and more pronounced than in the data, which may indicate that the thermal circuit of the bolometer is

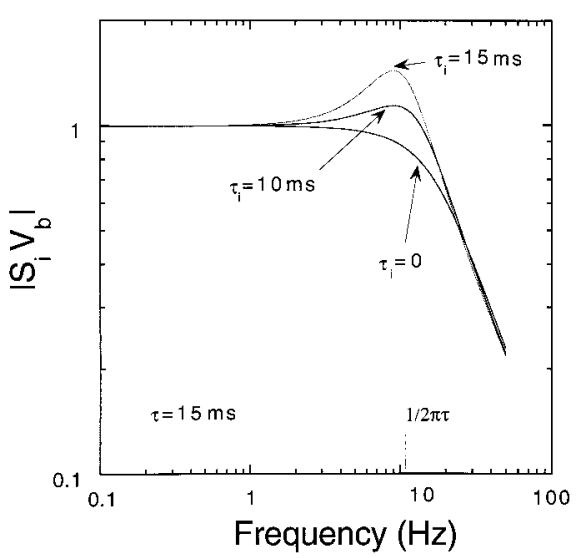

Fig. 10. Calculation of the effect of an internal time constant $\tau_{i}$ on the bolometer responsivity $\left|S_{i}(f) V_{b}\right|$. The model assumes that the bias power enters a thermometer with heat capacity $C_{i}$ and thermal conductance $C_{i} / \tau_{i}$ to the rest of the bolometer. The bolometer is biased so that $\tau \sim 15 \mathrm{~ms}(\mathscr{L}=170)$. For $\tau_{\mathrm{i}} \ll \tau, \mathrm{V}_{\mathrm{b}} \mathrm{S}_{\mathrm{i}}(\mathrm{f})$ has a Lorentzian roll off at $1 / 2 \pi \tau$. For values of $\tau_{i}$ comparable with $\tau$, there is a peak in $S_{i}(f)$ near $1 / 2 \pi \tau$, which causes a peak in the output noise arising from thermal fluctuations. This feature disappears as the gain is reduced.

more complex than that of our model. In general, the peak in responsivity should be most pronounced when the bolometer time constant is limited by the internal thermalization time, which is consistent with our observation on another VSB. ${ }^{18}$ A peak in noise was also observed with earlier measurements of a very fast VSB.4,5 A similar mechanism was hypothesized in which a peak in responsivity occurs when the effective time constant of the bolometer approaches the L/R time constant of the SQUID inductance and the bolometer resistance. However, this effect is unlikely to contribute to the peak in the noise spectrum of the current data sincethe L/R time constant is much smaller then the effective time constant.

\section{Conclusion}

We have fabricated a composite VSB with a Ti-film thermometer. The VSB has an average thermal conductance of $3.5 \times 10^{-10} \mathrm{~W} / \mathrm{K}$ and operates at 375 $\mathrm{mK}$ with a ${ }^{3} \mathrm{He}$ refrigerator. The largest value of $\alpha$ of our $\mathrm{Ti}$ thermometer was found to be $\sim 1000$. The measured $\tau_{0}=\mathrm{C} / \mathrm{G}$ of the tested VSB is approximately $2.6 \mathrm{~s}$. With ETF, the time constant is reduced by a factor of $1 /(\mathscr{L}+1)$ as predicted. The shortest observed response time is $\sim 13 \mathrm{~ms}$, which is 200 times faster than without ETF. Within the steep part of the resistive transition, the lowfrequency current responsivity is equal to $-1 / \mathrm{V}_{\mathrm{b}}$ within 1\%. The linear range of the VSB is 2 orders of magnitude wider than that could be achieved by a neutron-transmutation-doped Gebolometer operated with similar $\bar{G}$ and bias power. The measured noise includes a $1 / \mathrm{f}$ component for $\mathrm{f}<0.6 \mathrm{~Hz}$, which could be due to microphonics. For a bolometer resistance of $5 \Omega(\mathscr{L} \approx 20)$, the noise for $\mathrm{f}>0.6 \mathrm{~Hz}$ is close to that predicted from thermal fluctuation noise and J ohn- 
son noise. For lower resistance (higher gain) some excess noise is seen over a wide range of frequencies, with a suggestion of a broad peak near $1 / 2 \pi \tau$. Such an effect may be caused by a time delay in the propagation of heat from the thermal fluctuation to the thermometer.

For applications in which the thermal conductance of the weak link is limited by background power, the VSB should yield essentially the ideal NEP without the severe constraint on the heat capacity encountered in conventional bolometers. Alternatively, in applications in which a speed requirement governs the selection of $\mathrm{G}$ for a conventional bolometer, a substantial reduction in NEP should be possible with the VSB. In applications in which the background power changes substantially, thelargelinear range of a VSB can simplify the detector operation compared with that of conventional bolometers. The responsivity is independent of moderate variations of basetemperature and power loading, which means that calibration should be simpler and more accurate. These properties are of value in a wide range of applications from laboratory Fourier transform spectroscopy to infrared astronomy.

Finally, the VSB has advantages in ease of fabrication and implementation compared with conventional bolometers. Strong efforts to reduce the heat capacity are not necessary. Arrays of VSB's for astronomical observations can be manufactured with entirely photolithographic techniques in which, for example, silicon or silicon nitride isolation structures are used. SQUID amplifiers dissipate very low power $(\sim 1 \mathrm{nW})$ compared with fiel d-effect transistors and in principle can be operated on the same temperature stage.

The authors thank Tim Shaw, Thomas Lee, Gene Dansker, Roland Clarke, Sae Woo Nam, and Blas Cabrera for helpful discussions. This research was supported in part by the Director, Office of Energy Research, Office of Basic Energy Sciences, Materials Sciences Division of the U.S. Department of Energy under contract DE-AC03-76SF 00098. A. T. Lee was supported by the National Science Foundation through the Center for Particle Astrophysics (cooperative agreement AST 91-20005). S. F. Lee was also supported by a Rotary Club Fellowship from Rotary D-3460.

\section{References and Notes}

1. P. L. Richards, "Bolometers for infrared and millimeter waves," J . Appl. Phys. 76, 1-24 (1994).

2. S. T. Tanaka, A. C. Clapp, M. J . Devlin, N. Figueiredo, J . O. Gundersen, S. Hanany, V. V. Hristov, A. E. Lange, M. A. Lim, P. M. Lubin, P. R. Meinhold, P. L. Richards, G. F. Smoot, and J . Staren, "Measurements of anisotropy in the cosmic microwave background radiation at $0.5^{\circ}$ scales near the stars HR5127 and ф Herculis," Astrophys. J . L81, 468 (1996).

3. A. T. Lee, J . M. Gildemeister, S.-F. Lee, and P. L. Richards, "Voltage-biased high- $T_{C}$ superconducting infrared bolometer with strong electrothermal feedback," IEEE Trans. Appl. Supercond. 7, 2378-2381 (1997).

4. A. T. Lee, P. L. Richards, S. Nam, B. Cabrera, and K. D. Irwin, "A superconducting bol ometer with strong el ectrothermal feedback," Appl. Phys. Lett. 69, 1801-1803 (1996).

5. K. D. Irwin, "An application of electrothermal feedback for high resolution cryogenic particle detection," Appl. Phys. Lett. 66, 1998-2000 (1995).

6. This term is a modification of the effective thermal conductance defined in Ref. 1.

7. P. Horowitz and W. Hill, The Art of Electronics (Cambridge $U$ Press, Cambridge, UK, 1989), Chap. 4.

8. J. C. Mather, "Bolometer noise: nonequilibrium theory," Appl. Opt. 21, 1125-1129 (1982).

9. J . Clarke and T. Y. Hsiang, "Low-frequency noise in tin and lead films at the superconducting transition," Phys. Rev. B 13, 4790- 4800 (1976).

10. Y. B. Kim, C. F. Hempstead, and A. R. Strnad, "Resistive states of hard superconductors," Rev. Mod. Phys. 36, $43-45$ (1964).

11. Epoxy 907, Miller-Stephenson Chemical Company Inc., George Washington Hwy., Danbury, Conn. 06810.

12. H20E, Epoxy Technology Inc., 14 Fortune Drive, Billerica, Mass. 01821.

13. L. Duband, D. Alsop, A. Lange, and P. Kittel, "A rocket-borne ${ }^{3}$ He refrigerator," Adv. Cryog. Eng. 35, 1447-1456 (1989).

14. J . J . Bock, "Rocket-borne observation of singly ionized carbon $158 \mu \mathrm{m}$ emission from the diffuse interstellar medium," Ph. D. dissertation (University of California, Berkeley, Calif., 1994).

15. Model 50 SQUID and Model 550 Single Channel SQUID Controller, Quantum Design, 11578 Sorrento Valley Road, San Diego, Calif. 92121.

16. HP35660A Dynamic Signal Analyzer, Hewlett Packard Corporation, Everett, Wash. 98205.

17. This value was obtained by W. Holmes in a previous study.

18. A. T. Lee, S-F. Lee, J. M. Gildemeister, and P. L. Richards, "Voltage-biased superconducting bolometers for infrared and mm-wave astronomy," in Proceedings of the 7th International Workshop on Low Temperature Detectors LTD-7 (Max Planck Institute of Physics, Munich, Germany, 1997) available from urg@mppmu.mpg.de and at http://bolo.berkeley.edu. 